\title{
Hybrid treatment of massive pulmonary embolism by catheter-directed and surgical embolectomy
}

\author{
Arkadiusz Pietrasik ${ }^{1}$, Aleksandra Gąsecka ${ }^{1}$, Mateusz Leśniewski ${ }^{1}$, Dariusz Zieliński ${ }^{2}$, Szymon Darocha ${ }^{3}$, \\ Marcin Kurzyna ${ }^{3}$ \\ ${ }^{1} 1^{\text {st }}$ Chair and Department of Cardiology, Medical University of Warsaw, Warsaw, Poland \\ ${ }^{2}$ Department of Cardiac Surgery, Medicover Hospital, Warsaw, Poland \\ ${ }^{3}$ Department of Pulmonary Circulation, Thromboembolic Diseases and Cardiology, Centre of Postgraduate Medical Education, European \\ Health Centre Otwock, Poland
}

Adv Interv Cardiol 2021; 17, 2 (64): 236-238

DOI: https://doi.org/10.5114/aic.2021.107512

Pulmonary embolism (PE) is a life-threatening disease, responsible for up to 300000 deaths annually in the US, ranking among the leading causes of cardiovascular mortality [1]. Systemic thrombolytic therapy is recommended for patients with high-risk PE [2]. Yet, thrombolysis is contraindicated or has failed in numerous patients. Surgical or percutaneous catheter-directed therapies are alternative treatment options. We present a case of massive pulmonary embolism in a patient with high-risk PE, visualized during pulmonary angiography and extracted en bloc by surgical embolectomy.

A 53-year-old woman with a history of subarachnoid hemorrhage 30 days earlier was admitted to the Intensive Cardiac Care unit with high-risk PE (Pulmonary Embolism Severity Index class IV). The patient was consulted within the local Pulmonary Embolism Response Team (PERT) - regarding progressive hemodynamical deterioration, high risk of death and the absolute contraindication to systemic thrombolysis, the patient was referred for immediate percutaneous catheter-based intervention. Since no persistent bleeding was seen on the control head computed tomography, the consulting neurosurgeon supported the proposed interventional treatment.

In the catheterization laboratory, the patient presented with tachycardia of 120 beats/min, invasively measured aortic pressure (AoP) 91/53 mm Hg (mean: $65 \mathrm{~mm} \mathrm{Hg}$ ) and pulmonary arterial pressure (PAP) $50 / 22 \mathrm{~mm} \mathrm{Hg}$ (mean: $31 \mathrm{~mm} \mathrm{Hg}$ ). Pulmonary angiography demonstrated free-floating thrombi located in the proximal part and in all segmental branches of the right pulmonary artery, and within the inferior lobe branches on the left side (Figures 1 A, B). Percutaneous thrombectomy using the Cleaner XT Rotational Thrombectomy System was performed in the proximal part of the right pulmonary artery. Due to suboptimal results of rotational defragmentation, thrombi were additionally aspired using the Indigo System 8F Penumbra (Figure $1 \mathrm{C}$ ). The percutaneous procedure allowed for transient hemodynamic stabilization, as indicated by the heart rate of 90 beats/ min, AoP 126/82 mm Hg (mean: $95 \mathrm{~mm} \mathrm{Hg}$ ) and PAP 46/ $19 \mathrm{~mm} \mathrm{Hg}$ (mean: $28 \mathrm{~mm} \mathrm{Hg}$ ). However, the clinical result was insufficient, since the patient still required substantial inotropic support. One day later, surgical embolectomy was performed, enabling extraction of fresh thrombus and well-organized thrombotic lesions from both right and left pulmonary arteries (Figure $1 \mathrm{D}$ ), resulting in a further decrease in PAP to $25 / 13 \mathrm{~mm} \mathrm{Hg}$ (mean: $17 \mathrm{~mm} \mathrm{Hg}$ ). The thrombi extracted from the right pulmonary artery were fragmented due to the preceding mechanical thrombectomy, whereas the thrombi from the left pulmonary artery were removed en bloc.

The follow-up echocardiography performed a week later demonstrated normal left ventricle function and no right ventricle overload. Following the in-hospital rehabilitation, the patient presented with only mild cognitive dysfunction and was mobile, if assisted.

Surgical embolectomy is the preferred form of treatment in patients with PE and contraindications to systemic thrombolysis due to the possibility of en bloc removal of the thrombi and favorable results in the recent trials [2]. However, patients with extensive proximal thrombi may require combined percutaneous and surgical treatment [3]. In our patient, the organized and fibrotic thrombi removed en bloc might suggest that the

\section{Corresponding author:}

Aleksandra Gąsecka MD, PhD, $1^{\text {st }}$ Chair and Department of Cardiology, Medical University of Warsaw, Warsaw, Poland, phone: +48518343599, e-mail: aleksandra.gasecka@wum.edu.pl

Received: 8.02.2021, accepted: 6.03.2021. 

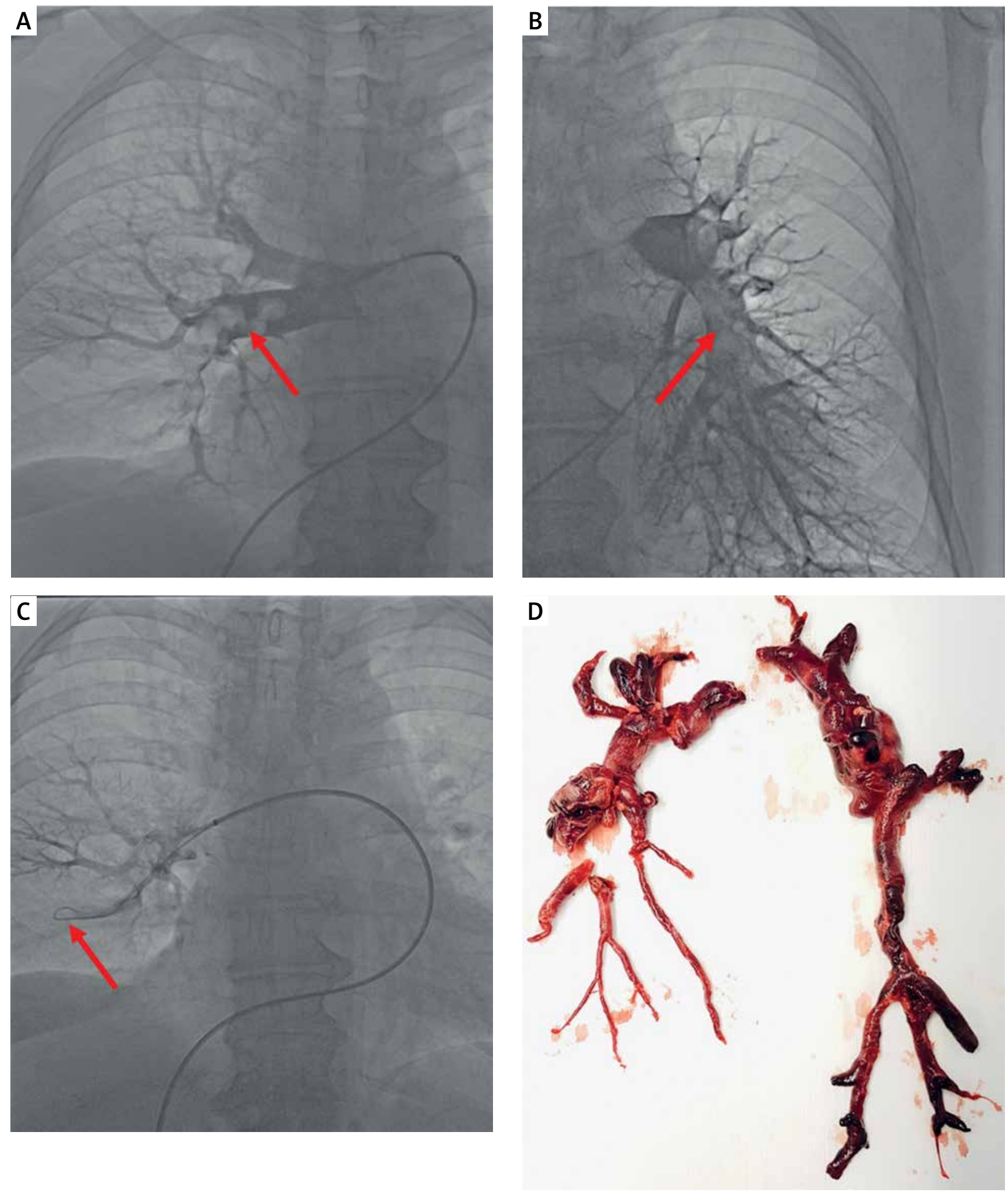

Figure 1. A, B - Selective pulmonary angiogram revealing free-floating thrombi masses (red arrows) located in the proximal part and in all segmental branches of the right pulmonary artery (A) and within the inferior lobe branches of the left pulmonary artery (B). C - Subsequent phases of percutaneous mechanical thrombectomy with the Cleaner XT catheter followed by aspiration of the thrombi from the right pulmonary artery with the Indigo System 8F Penumbra (red arrow). D - Surgical specimen extracted during surgical embolectomy, demonstrating fragmentated thrombi from the right pulmonary artery and en bloc thrombi from the left pulmonary artery 
acute PE was overlapping with the pre-existing chronic thromboembolic lesions. Well-organized, real-time communication within the local PERT is crucial to enhance decision-making and facilitate immediate treatment [4].

\section{Conflict of interest}

The authors declare no conflict of interest.

\section{References}

1. Wendelboe AM, Raskob GE. Global burden of thrombosis: epidemiologic aspects. Circ Res 2016; 118: 1340-7.

2. Konstantinides SV, Meyer G, Becattini C, et al. 2019 ESC Guidelines for the diagnosis and management of acute pulmonary embolism developed in collaboration with the European Respiratory Society (ERS). Eur Heart J 2020; 41: 543-603.

3. Kurzyna M, Pietrasik A, Opolski G, Torbicki A. Contemporary methods for the treatment of pulmonary embolism - is it primetime for percutaneous interventions? Kardiol Pol 2017; 75: 1161-70.

4. Dudzinski DM, Piazza G. Multidisciplinary pulmonary embolism response teams. Circulation 2016; 133: 98-103. 